\title{
A review on humanoid robotics in healthcare
}

\author{
Azeta Joseph, Bolu Christian, Abioye A. Abiodun and Festus Oyawale \\ ${ }^{1}$ Department of Mechanical Engineering, Covenant University, Ota, Nigeria.
}

\begin{abstract}
Humanoid robots have evolved over the years and today it is in many different areas of applications, from homecare to social care and healthcare robotics. This paper deals with a brief overview of the current and potential applications of humanoid robotics in healthcare settings. We present a comprehensive contextualization of humanoid robots in healthcare by identifying and characterizing active research activities on humanoid robot that can work interactively and effectively with humans so as to fill some identified gaps in current healthcare deficiency.
\end{abstract}

\section{Introduction}

The use of robots for surgery has given rise to a large number of new methods which has led to the emergence of a wide variety of robots for use in the medical domain. [1] Points out numerous research areas in the application of robotics in healthcare settings. Such as robotic surgical Systems [2, 3], laparoscopy surgery and tele-rounding robots [4], robot-assisted rehabilitation [5-7], caregiver and patient's assistants [8-10], robotic applications in dentistry, bio-prosthetic [11]. The list continues as the technology advances and even more applications of robot in healthcare could be envisaged.

Humanoid robots that could be used to remotely carry out tasks are also very important. Such robots are endowed with human capabilities to assist caregivers and patients particularly in contaminated environments. These remotely operated robots are to possess certain characteristics for human robot interaction. However, tele-operated semi-autonomous robots can be used to perform assisted healthcare tasks during outbreaks which would reduce the time personnel need to spend in dangerous contaminated areas while putting on their personal protective equipment in high temperature and humid conditions particularly in the West African region. Furthermore [12] highlighted some healthcare robots that have been developed for similar fields of application [13, 12]. The robot Cody is able to wash human limbs autonomously and an evaluation of the patient's system showed that acceptance strongly depends on the interpretation of robot intention [14]. Other robots serve as rehabilitation [15], the nurse assisting lifting of patients [16] or even blood sampling [17].

While research on humanoid robots for general or supportive patients care particularly for disease containment has hit the ground running in other developed countries due to the recent outbreak of Ebola virus disease that shook the world it is also important for those affected directly to find a solution to their problem. We propose a broad overview of some of the current and potential applications of robotics in health care settings and we carefully selected some robots for patients care.

\section{Literature Review}

Table 1. Summary of different application of robot for general and supportive care [13].

\begin{tabular}{lll}
\hline Author & Robot & Description \\
\hline$[18]$ & ARMAR III & $\begin{array}{l}\text { Developed to support tasks in 'human-centered' environments to include } \\
\text { households. Upper torso is humanoid in design. Designed to interact with humans } \\
\text { and manipulate objects in the environment. }\end{array}$ \\
[19] & Care-O-Bot 3 & $\begin{array}{l}\text { Mobile robot assistant designed to help humans in everyday environments. } \\
\text { Includes an arm and gripper for manipulation of objects, a tray for carrying and } \\
\text { transferring objects, and a flexible torso enabling butler-like gestures such as } \\
\text { bowing and nodding. }\end{array}$ \\
[20] & Cody & $\begin{array}{l}\text { Robot assistant developed to help caregivers with patient hygiene, specifically bed } \\
\text { baths. Uses a compliant arm and gentle force to perform 'wiping motions' similar } \\
\text { to those used during bed baths. } \\
\text { Mobile humanoid robot design to support tasks in human environments. Capable }\end{array}$
\end{tabular}


[9] RIBA High load capacity robot designed to lift and transfer patients.

[22] RIBA Designed in appearance similar to a giant teddy bear. Carries patients in 'humantype' arms. Equipped with tactile sensors to detect patient's contact position, allowing motion adjustment to be made accordingly.

[16] Robotic Nursing Designed to help caregivers with physically demanding tasks such as lifting and Assistant transferring patients. Upper torso is humanoid in design.

[23] Hair-Washing Robot assistant designed to help caregivers wash patient's hair.

Robot
ASIMO

[25] ROSE

Human-size humanoid robot. Capabilities include walking, running, and climbing stairs, carrying objects, opening doors, and pushing carts.

Tele-operated robot for home care applications. Capable of performing tasks such as grabbing and placing objects, opening doors, and cleaning.

\section{Characteristics of Humanoid robot}

\subsection{Vision system}

Visual perception is fundamental to most robotics systems working in human environments, it is also essential to a wide variety of tasks such as manipulation, tracking, human-robot interaction [26]. [27] Developed a simple and affordable vision-based robotic system for the identification of the Euclidean position of red spheres that emulate ripe tomatoes. This is done by using a RGB-D sensor in a fixed position, together with a 5 DOF manipulator. Robotics should follow the condition and perceive the surroundings to achieve the goal. Due to perception, robots check their state of joints by using encoders or sensors. Robots developed nowadays are covered with force-sensitive skins. Many humanoid robots use senses like laser rangefinders or ultrasonic distance sensors. They also use cameras, to enable them to focus their attention towards specific objects and screens to interpret the images.

\subsection{Manipulation tasks}

Humans have the ability to move their hands almost 30 DOF [28]. Some of the humanoid robots that were built cannot hold the unknown objects from the environment like humans. The reason behind this is lack of learning ability to perceive and sense the new objects. Improvements were needed so that they could hold the things and sense them by touching [28]. Also learning from demonstration can be used to train soft robotic hands to perform dexterous manipulation tasks. The ReMeDi robot led to the design of a light cable-driven manipulator [29] which then advanced to the construction of a large SCARA type manipulator with 6 DOF which is used for palpation [12].

\subsection{Sensing behavior}

There are different types of sensors used in humanoid robots. But [28] focused on specific on sensors used to grasp the objects means arm's sensors. Sometimes arms are small so according to them, sensors must be small according to the joints. For loop gesture of hand, there should be a measurement of every joint. [28] Highlighted that from the many approaches that were used for this [30] gave concept of using plastic potentiometer at ever finger's joints. Another technique by [31] gave that linear potentiometers at wrist joint to calculate the angles of joints. But this idea was flopped due to lack of precision cause of friction of tendons. Most useful idea was given by [32].

\subsection{Mobile Platform}

Humanoid robots have to be flexible enough for easy maneuvering. While fixed robots will always find a place in manufacturing, humanoid robots with mobile base promises additional flexibility to end-user in new applications. These applications include caregiver and patients assistance, medical and surgical uses as well as security. [12] Propose two types of motion for the mobile base, the short distance motion which cater for the robots positioning next to the patient to obtain an appropriate position area for the robot arm and a long distance motion which involves movement between the robot parking lot and the examination room.

\section{Applications of Humanoid Robotics in Healthcare}

\subsection{Tele-Healthcare}

A Tele-operated Service Robot is a robot that is controlled by a human being from a distance and performs tasks typically in uncontrolled environments [25]. Tele-operation enables an operator, to act remotely as if the operator was on the spot, by for instance copying the manipulations of the operator at a distance. An example is the Da Vinci Robot used for medical surgery [25].

The use of tele-operated humanoid robots in healthcare represents an exciting opportunity to help doctors, nurses and patients to ease the high risk of 
infectious disease transfer [33]. [34] Investigated the use of teleoperated robots to allow healthcare workers to perform some of their duties at a safe distance from the infected patients. The result suggest that patients will trust the robot less when the operation is unseen than when the operation is visible. More investigation needs to be carried out to know the extent of the effect. However, the design of the Tele-operated Service Robot [25] can be adapted for the development of a healthcare robot for the general task of supportive patients care.

\subsection{Humanoid Robot for Pain Relief}

Humanoid robots are used to implement technopsychological distraction for children in order to reduce their pain as a result of stress and anxiety during a medical procedure. Studies carried out by [35-37] all point to the fact that children are more likely to smile when encountering medical procedures with a robot. The aim is to turn children's attention away from the pain of the needle toward an amusing activity. [38, 37]. States the principles of attentional capacity theory, that the distraction stimulus must be stronger than the pain stimulus to gain the child's attention. Although, music and cartoons have shown effectiveness in reducing pain and anxiety among children undergoing a variety of medical procedures $[39,40,36]$. It would appear that these distractions are not always strong enough to turn children's attention away from the pain. It is now believed that multisensory strategies, which combine visual, auditory, and tactile senses, may have a greater impact on pain than single-sensory strategies $[39,36]$. Given the mixed results mentioned above, it stands to reason that stronger and more engaging forms of distraction, which invite the child to engage in an activity, may be necessary for medical procedures.

In addition to other interventions, we suggests that techno-psychological distraction for children for management of pediatric pain be considered. When programmed with humanistic characteristics and to execute psychological strategies, a humanoid robot shows promise of reducing procedural pain and distress in children [37],

\subsection{Humanoid Robot for Aging Population}

In a society where there is a rise in the disabled and aging population, there is a strong demand for robotics to tackle problems that arise from their inabilities to relate effectively with their environment. Robots for executing patient-transfer tasks are needed in nursing care facilities and hospitals, [9] developed a new prototype robot named RIBA with human-type arms which was designed to perform heavy physical tasks requiring human contact, the robot was able to transfer a human from a bed to a wheelchair and back. The caregiver can intuitively give instructions to RIBA through tactile sensors using a newly proposed method named tactile guidance. RIBA was developed to cope the difficulties encountered previously by RI-MAN [41, 42].

\section{Conclusion}

More interest are now being directed towords robotics in healthcare because of the successes robots can bring as it has been in other domain. Still, we have not seemed to have been able to appreciably utilize this technology in developing countries for effective healthcare delivery. Healthcare needs a pathfinder solution in which the investment and risk of robotics applications are small. However, There are a number of exciting advances in robotics in recent years, which points to a fruitful future. Humanoid robotics in healthcare settings is rapidly evolving. We examined the state-of-the-art of the emerging field of the current and potential applications of robotics in healthcare.

\section{References}

1. Krzysztof Arent, Mateusz Cholewiński, Łukasz Chojnacki, Wojciech Domski, Micha łDrwięga, Janusz Jakubiak, Mariusz Janiak, Bogdan Kreczmer, Adam Kurnicki, Bartłomiej Stańczyk, D. S.-S. Selected Topics in Design And Application of a Robot For Remote medical Examination With use Of Ultrasonography And Auscultation from The Perspective of The Remedi Project. Journal of Automation, Mobile Robotics \& Intelligent Systems, VOLUME11(N॰2). (2017).

2. Z. Nawrat. Postepy robotyki. Przemystowe $i$ medyczne systemy robotyczne, chapter Polski robot kardiochirurgiczny, 275-284. Wydawnictwa Komunikacji i Łączności, (2005).

3. "The da Vinci Surgical System, the manufacturer website". http://www.intuitivesurgical.

4. M. Iftikhar, M. J. Majid, M. Muralindran, G. Thayabaren, R. Vigneswaran, and T. T. K. Brendan, "Otorob: Robot for orthopaedic surgeon roboscope: Non-interventional medical robot for telerounding". In: Proceedings of the 5th International Conference on Bioinformatics and Biomedical Engineering, 1 - 5. (2011).

5. S. Balasubramanian, J. Klein, and E. Burdet. Robotassisted rehabilitation of hand function. Curr Opin Neurol, (2010).

6. T. Nef, M. Mihelj, and R. Riener, "Armin: a robot for patient-cooperative arm therapy", Medical and Biological Engineering and Computing, vol. 45, 887-900. (2007).

7. M. Kaczmarski and G. Granosik, "Rehabilitation robot rrh1", The Archive of mechanical Engineering, vol. 58, no. 1, (2011).

8. M. Mariappan, T. Ganesan, V. Ramu, and M. Iftikhar. Intelligent Robotics and Applications, chapter Safety System and Navigation for Orthopaedic Robot (OTOROB), 358 - 367. Springer, (2011).

9. T. Mukai, S. Hirano, H. Nakashima, Y. Kato, Y. Sakaida, S. Guo, and S. Hosoe, "Development of a nursing-care assistant robot riba that can lift a human in its arms". In: Proceedings of the IEEE/RSJ International Conference on Intelligent Robots and Systems, (2010). 
10. C. Urdiales, M. Fernandez-Carmona, J. M. Peula, R. Annicchiaricco, F. Sandoval, \& C. Caltagirone. Problemy robotyki, chapter A collaborative control scheme for haptics-based blind wheelchair driving. Oficyna Wydawnicza Politechniki Warszawskiej, (2010).

11. J. T. Belter, J. L. Segil, A. M. Dollar, and R. F. Weir, "Mechanical design and performance specifications of anthropomorphic prosthetic hands: A review", Journal of Rehabilitation Research \& Development, vol. 50, no. 5, 599-618. (2013).

12. Panzirsch, M., Weber, B., Rubio, L., Coloma, S., Ferre, M., \& Artigas, J. Tele-healthcare with humanoid robots: A user study on the evaluation of force feedback effects. 2017 IEEE World Haptics Conference (WHC), (June), 245-250. (2017). https://doi.org/10.1109/WHC.2017.7989909

13. K. Doelling, J. Shin, and D. O. Popa, "Service robotics for the home: a state of the art review,"in Int. Conf. on Pervasive Technologies Related to Assistive Environments. ACM, p. 35. (2014).

14. T. L. Chen, C.-H. A. King, A. L. Thomaz, and C. C. Kemp, "An investigation of responses to robotinitiated touch in a nursing context, "Int. Journal of Social Robotics, vol. 6, no. 1, pp. 141-161, (2014).

15. Barrett Medical, "Proficio," http:/www.barrettmedical.com/, accessed: 2016-0219.

16. J. Hu, A. Edsinger, Y.-J. Lim, N. Donaldson, M. Solano, A. Solochek, and R. Marchessault, "An advanced medical robotic system augmenting healthcare capabilities-robotic nursing assistant," in Int. Conf. on Robotics and Automation. IEEE, pp. 6264-6269. (2011).

17. A. I. Chen, M. L. Balter, T. J. Maguire, and M. L. Yarmush, "Realtime needle steering in response to rolling vein deformation by a 9dof image-guided autonomous venipuncture robot," in Int. Conf. on Intelligent Robots and Systems. IEEE, pp. 26332638. (2015).

18. Asfour, T., et al. ARMAR-III: An integrated humanoid platform for sensory-motor control. IEEERAS International Conference on Humanoid Robots. (Genova, Italy, Dec. 4-6), 169-175. (2006).

19. Reiser, U., et al. Care-O-bot $\AA 3$ - creating a product vision for service robot applications by integrating design and technology. IEEE/RSJ International Conference on Intelligent Robots and Systems. (St. Louis, USA, Oct. 11-15), 1992-1998. (2009).

20. King, C.-H., et al. Towards an assistive robot that autonomously performs bed baths for patient hygiene. IEEE/RSJ International Conference on Intelligent Robots and Systems. (Taipei, Taiwan, Oct. 18-22), 319-324. (2010).

21. Cousins, S., ROS on the PR2. IEEE Robotics \& Automation Magazine. 1, 3 23-25. (Sept 2010).

22. Mukai, T., et al.. Tactile-based motion adjustment for the nursing-care assistant robot RIBA. IEEE International Conference on Robotics and Automation. (Shanghai, China, May 9-13), 5435 5441. (2011).
23. Hirose, T., et al. Development of hair-washing robot equipped with scrubbing fingers. IEEE International Conference on Robotics and Automation. (Saint Paul, USA, May 14-18, 2012), 1970-1975. (2012).

24. American Honda Motor Co. Inc. History of ASIMO. Retrieved Jan. 10, (2014) from http://asimo.honda.com/asimo-history/.

25. Osch, M. V., Bera, D., Hee, K. V., Koks, Y., \& Zeegers, H. Tele-operated service robots:ROSE. Automation in Construction 39, 152-160. (2014).

26. Sean Ryan Fanello, Carlo Ciliberto, Nicoletta Noceti, Giorgio Metta, F. O. Visual recognition for humanoid robots. Robotics and Autonomous Systems, 91, 151-168.

(2017).

https://doi.org/10.1016/j.robot.2016.10.001

27. García-Luna, F., \& Morales-Díaz, A. Towards an artificial vision-robotic system for tomato identification. IFAC-PapersOnLine, 49(16), 365-370. (2016). https://doi.org/10.1016/j.ifacol.2016.10.067

28. Rabbia Mahum *, Faisal Shafique Butt, Kashif Ayyub, Seema Islam, Marriam Nawaz, D. A. International Journal of Advanced and Applied Sciences. International Journal of Advanced and Applied Sciences, 4(2), 83-90. (2017). Retrieved from http://science-gate.com/IJAAS/Articles/2016-312/14 2016-3-12-pp.106-112.pdf

29. A. Kurnicki, B. Stanczyk, and B. Kania, "Manipulator development for telediagnostics". In: Proceedings of the International Conference on Mechatronics and Robotics, Structural Analysis (MEROSTA2014), 214 -218. (2014).

30. Butterfass J, Grebenstein M, Liu H, and Hirzinger G. DLR-Hand II: Next generation of a dextrous robot hand. In the IEEE International Conference on Robotics and Automation (ICRA), IEEE, Seoul, South Korea, 1: 109-114. (2001). https://doi.org/10. 1109/ROBOT.2001.932538

31. Chua PY, Caldwell DG, Bezdicek M, Gray JO, and Davis S. Tele-operated high speed anthropomorphic dextrous hands with object shape and texture identification. In the IEEE/RSJ International Conference on Intelligent Robots and Systems, IEEE, Beijing, China: 4018-4023. (2006). https://doi.org/10.1109/ IROS.2006.281861

32. Behnke, S. Humanoid robots-from fiction to reality?. In the Künstliche Intelligenz Heft, 4, 5-9. (2008). Available online at: https://www.ais.unibonn.de/papers/KI08 Behnke.pdf

33. Riek, L. D. Healthcare Robotics. (2017). Retrieved from http://arxiv.org/abs/1704.03931

34. Kraft, K., \& Smart, W. D. Seeing is comforting: Effects of teleoperator visibility in robot-mediated health care. ACM/IEEE International Conference on Human-Robot Interaction, 2016-April, 11-18. (2016). https://doi.org/10.1109/HRI.2016.7451728

35. Yasemin, M., \& Kasimo, Y. Management of dental anxiety in children using robots Reduction of Dental Anxiety and Pain in Children using Robots, (May), 327-332. https://doi.org/10.1109/SIU.2016.7495721
(2016). 
36. Beran, T. N., Ramirez-Serrano, A., Vanderkooi, O. G., \& Kuhn, S. Humanoid robotics in health care: An exploration of children's and parents' emotional reactions. Journal of Health Psychology, 20(7), 984989.

(2015).

https://doi.org/10.1177/1359105313504794

37. Beran, T. N., Ramirez-Serrano, A., Vanderkooi, O. G., \& Kuhn, S. Reducing children's pain and distress towards flu vaccinations: A novel and effective application of humanoid robotics. Vaccine, 31(25), 2772-2777.

(2013).

https://doi.org/10.1016/j.vaccine.2013.03.056

38. [38] McCaul KD, Malott JM. Distraction and coping with pain. Psychol Bull; 95(3):516. (1984).

39. DeMore M, Cohen L. Distraction for pediatric immunization pain: a critical review. J Clin Psychol Med Settings; 12(4):281-91. (2005)
40. Klassen, JA., Liang, Y., Tjosvold, L., Klassen, TP., Hartling, L. "Music for pain and anxiety in children undergoing medical procedures: a systematic review of randomized controlled trials", Ambulatory Pediatrics, 8, 117-128. (2008).

41. M. Onishi, Z. W. Luo, T. Odashima, S. Hirano, K. Tahara, and T. Mukai, "Generation of Human Care Behaviors by Human-Interactive Robot RI-MAN," in Proc. IEEE International Conference on Robotics and Automation (ICRA), pp. 3128-3129, (2007).

42. T. Mukai, M. Onishi, T. Odashima, S. Hirano, and Z. W. Luo, "Development of the Tactile Sensor System of a Human- Interactive Robot 'RI-MAN',' IEEE Trans. on Robotics, Vol.24, No.2, pp.505- 512, (2008 